\title{
Elevated Temperature, In Situ Micromechanical Characterization of a High Temperature Ternary Shape Memory Alloy
}

\author{
J.M. WHEELER $\odot,{ }^{1,2,5}$ C. NIEDERBERGER, ${ }^{1}$ R. RAGHAVAN, ${ }^{1,3}$ \\ G. THOMPSON, ${ }^{4}$ M. WEAVER,${ }^{4}$ and J. MICHLER ${ }^{1}$
}

1.-Laboratory for Mechanics of Materials and Nanostructures, Empa, Swiss Federal Laboratories for Materials Science and Technology, Feuerwerkerstrasse 39, 3602 Thun, Switzerland. 2.-Laboratory for Nanometallurgy, Department of Materials Science, ETH Zürich, VladimirPrelog-Weg 5, 8093 Zurich, Switzerland. 3.-Structure and Nano-/Micromechanics of Materials, Max-Planck-Institut für Eisenforschung GmbH, Max-Planck-Strasse 1, 40237 Düsseldorf, Germany. 4.-Department of Metallurgical and Materials Engineering, The University of Alabama, Tuscaloosa, AL, USA. 5.—e-mail: Jeff.wheeler@mat.ethz.ch

The microthermomechanical behavior of a precipitation-hardenable Ni-48Ti25Pd (at.\%) shape memory alloy has been investigated as a function of temperature. Micropillars were fabricated within a large $\langle 145\rangle$-oriented grain and compressed in situ in the SEM at elevated temperatures corresponding to the martensite and austenite phase transformation temperatures. The precipitation-strengthened alloys exhibited stable pseudoelastic behavior with little or no residual strains when near the transformation temperatures. In the plastic regime, slip was observed to occur via pencil glide, circumventing the fine scale precipitates along multiple slip planes.

\section{INTRODUCTION}

$\mathrm{Ni}$-Ti-based alloys have been extensively studied and are the only successfully commercialized shape memory materials because of their excellent balance of physical, mechanical, and functional properties. ${ }^{1,2}$ Much of the research related specifically to shape memory behavior has focused on the Tirich and equiatomic $\mathrm{Ni}-\mathrm{Ti}$ compositions because these alloys have higher transformation temperatures $\left(\sim 90-100^{\circ} \mathrm{C}\right)^{3,4}$ as compared to Ni-rich binary alloys, where transformation temperatures can be well below $27^{\circ} \mathrm{C} .^{5}$ In recent years, ternary and quaternary alloying additions, in particular $\mathrm{Pd}$ and/or Pt, have been used to increase the transformation temperatures of $\mathrm{NiTi}$ alloys above $100^{\circ} \mathrm{C}^{1-5}$ Specific to the Ni-rich alloys, these additions have been found to promote the formation of a ternary P-phase precipitate, which resulted in increased transformation temperatures. The precipitate, which is monoclinic and Ni-rich, depletes the surrounding matrix phase of $\mathrm{Ni}$ resulting in a Ti-rich matrix with a higher transformation temperature. ${ }^{6,7}$ The precipitates also act to strengthen the matrix against slip, producing more stable pseudoelastic and shape memory behaviors. ${ }^{7}$

However, as many applications of shape memory alloys, such as surgical implants or micro-actuators, rely on small length scale performance, the mechanical behavior of these materials on small scales is of interest. Previous in situ, micromechanical studies ${ }^{8,9}$ of shape memory have largely focused on these Ni-rich binary alloys, since their low transformation temperatures allow them to be pseudoelastic at ambient temperature. New in situ, elevated temperature nano-mechanical testing techniques, ${ }^{10,11}$ however, allow investigation of the mechanical response of a wider range of alloys with elevated temperature transformations. High temperature micro-compression testing allows us to understand the phase transformations that are associated with these new types of alloys. Such insights could indicate the stability at temperature under load. To date, this Ni-Ti-Pt/Pd alloy has not been extensively studied for its psuedoelastic 
behavior or at small length scales, which would complement the prior shape memory alloy (SMA) studies. This paper will address this deficiency.

\section{EXPERIMENTAL PROCEDURE}

\section{Material Production and Characterization}

An alloy ingot with nominal composition of Ni48Ti-25Pd (at.\%) was prepared by vacuum induction melting using a graphite crucible under a slight partial pressure of argon and casted into a copper mold of dimension of approximately $25 \mathrm{~mm}$ diameter by $102 \mathrm{~mm}$ in length. The ingot was homogenized in vacuum for $72 \mathrm{~h}$ at $1050^{\circ} \mathrm{C}$ followed by extrusion at $900^{\circ} \mathrm{C}$ with an area reduction ratio of $7: 1$. An approximately $10 \mathrm{~mm}$ diameter $\times 1 \mathrm{~mm}$ thick disc cut from the extruded bar was then directly subjected to a heat treatment at $400^{\circ} \mathrm{C}$ for $100 \mathrm{~h}$, which has previously been reported to promote the precipitation of the P-phase. ${ }^{7}$ During the aging process, the specimen was wrapped with Ta foil and Ar was flowed over it to mitigate oxidation. Differential scanning calorimetry (DSC) was carried out in a TA Instruments DSC Q1000 at a heating and cooling rate of $10^{\circ} \mathrm{C} / \mathrm{min}$ to characterize the transformation temperatures. Post-aging, the specimen was metallographically prepared to a final surface polish using a $0.25-\mu \mathrm{m}$ diamond suspension. A $500 \times 500 \mu \mathrm{m}$ region microstructure of the sample was then examined using electron backscattered diffraction (EBSD) with a step size of $2 \mu \mathrm{m}$ in using a Zeiss DSM962 scanning electron microscope (SEM) at $20 \mathrm{kV}$ accelerating voltage. A custom heating system was employed to perform EBSD at elevated temperatures: $25^{\circ} \mathrm{C}, 113^{\circ} \mathrm{C}, 134^{\circ} \mathrm{C}$, and $150^{\circ} \mathrm{C}$, respectively.

\section{Microcompression Testing}

Square micropillars with equivalent diameters of 3-4 $\mu \mathrm{m}$ and aspect ratios of 3-3.5 were fabricated within a large single grain identified by EBSD using a Ga ion beam at an accelerating voltage of $30 \mathrm{kV}$ in a Tescan Vela focused ion beam (FIB) instrument. Initially, high currents of $\sim 3 \mathrm{nA}$ were used to mill craters around the micropillars with larger than targeted diameters, which allowed clear viewing of the entire pillar length during compression. Next, the desired micropillar dimensions were achieved by polishing the coarse micropillars with lower ion currents ranging from $1 \mathrm{nA}$ to $120 \mathrm{pA}$, which in turn minimizes implantation damage. The micropillars were imaged using a Hitachi S4800 high-resolution scanning electron microscope (HRSEM) after FIB machining and after compression at elevated temperatures.

Microcompression testing was performed in situ inside a Zeiss DSM 962 SEM with an Alemnis SEM Indenter modified for high-temperature operation. ${ }^{11}$ Thermal drift in this system is minimized at each testing temperature by both a temperature shift ${ }^{12}$ and displacement drift ${ }^{13}$ tuning prior to compression testing. To ensure the accuracy of the surface temperatures tested, the indenter tip temperature was calibrated using a procedure described previously. ${ }^{12,14}$ Micropillars were compressed at a displacement rate appropriate to generate a $5 \times 10^{-4} \mathrm{~s}^{-1}$ strain rate for the heights of the pillars. To study the strain limits of the pseudoelastic behavior, cyclic microcompression using multiple load-unload-reload cycles was performed at each temperature.

\section{RESULTS AND DISCUSSION}

\section{Characterization}

Figure 1 shows the DSC trace obtained from the aged specimen. An endothermic peak for the B19 $\rightarrow$ B2 transformation was observed on heating, with a peak temperature of approximately $125^{\circ} \mathrm{C}$. Two exothermic peaks, Fig. 1, show a multi-step martensitic transformation on cooling with a martensite start, $M_{\mathrm{s}}$, and temperature of $100^{\circ} \mathrm{C}$. The multi-step martensitic transformation resulted from local stress and compositional inhomogeneities near P-phase precipitates within the alloy in contrast to the bulk matrix away from the precipitates. This also accounts for the shift of the peaks associated with the martensitic transformation as a function of aging. ${ }^{7}$

Figure 2 shows two EBSD grain orientation maps taken at $25^{\circ} \mathrm{C}$ and $150^{\circ} \mathrm{C}$. Some elongation is observed in the vertical direction due to thermal drift occurring during the elevated temperature mapping. This was expected, since the sample mount was not water-cooled. This allowed some thermal expansion to occur within the sample mount due to heat flow. The thermal expansion results in a shift in position of the sample during the scanning process. However, other than this distortion, no significant difference was observed in the EBSD maps at any of the temperatures. For this reason, the EBSD maps from $113^{\circ} \mathrm{C}$ to $134^{\circ} \mathrm{C}$ are not shown.

In both maps, the grain size distribution was found to be strongly bimodal with several larger $(>100 \mu \mathrm{m})$ [110]-and [001]-textured grains within

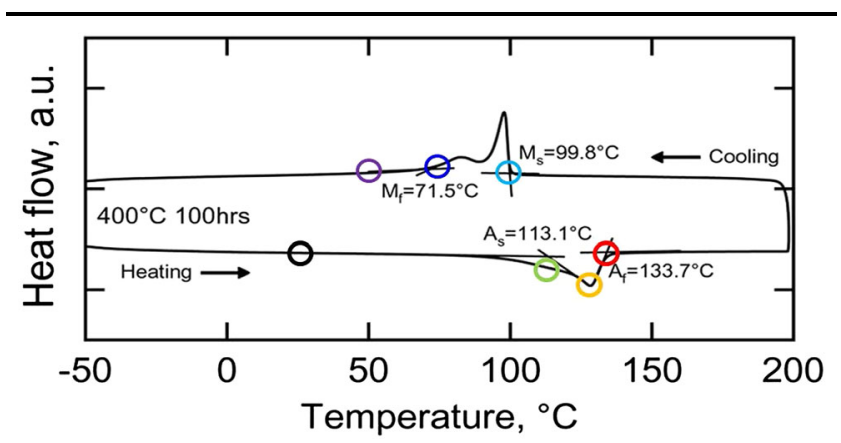

Fig. 1. DSC trace for the Ni-48Ti-25Pd alloy after aging at $400^{\circ} \mathrm{C}$ for $100 \mathrm{~h}$ (after Sasaki et al. ${ }^{7}$ ) with temperatures of interest highlighted. 

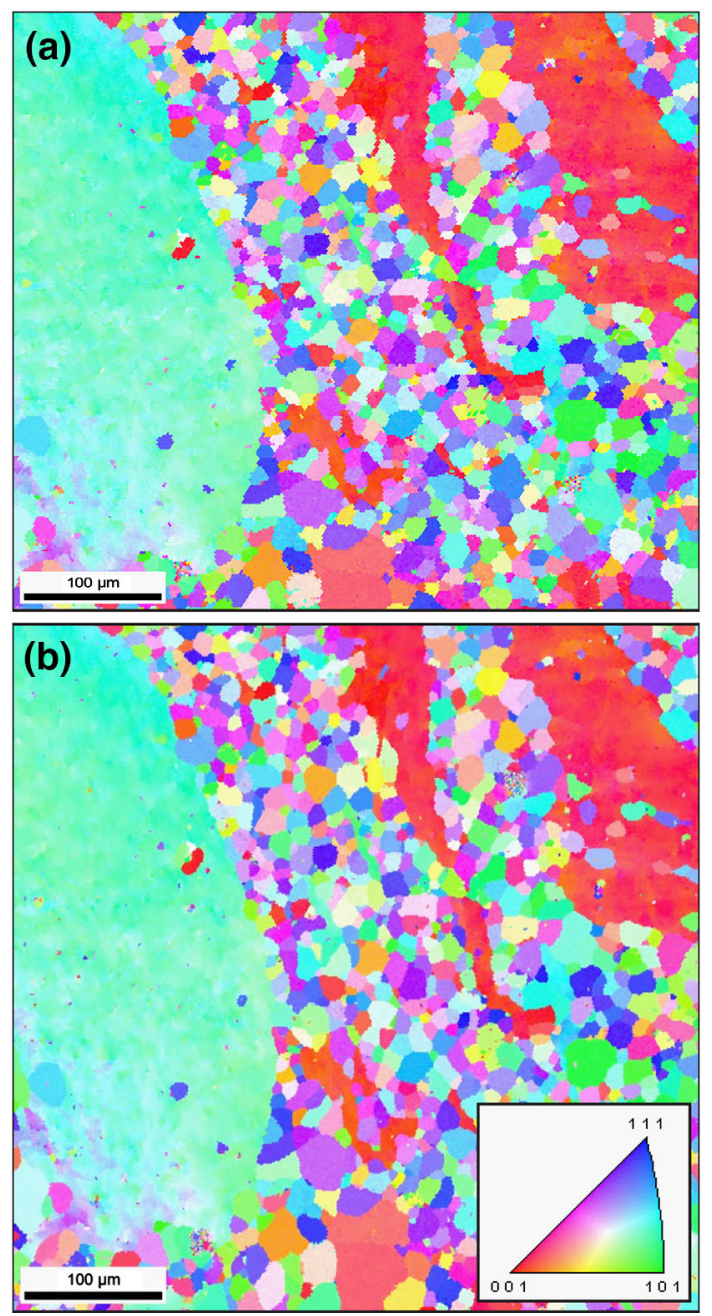

Fig. 2. EBSD inverse pole figure maps at (a) $25^{\circ} \mathrm{C}$ and (b) $150^{\circ} \mathrm{C}$.

a randomly oriented finer $(\sim 20 \mu \mathrm{m})$ grain structure. Coarse titanium carbide (TiC) precipitates were also observed throughout the matrix (most easily visible in Fig. 2 as very small, $1-2 \mu \mathrm{m}$ grains within the larger green grain to the left) and are a result of alloy processing in a graphite crucible. Their composition $(50.5 \% \mathrm{Ti}$ and $49.5 \% \mathrm{C}$ ) was confirmed using energy-dispersive x-ray spectroscopy (EDS).

Only the parent austenite phase was observed in all cases. The lack of observation of the martensite phase is attributed to the martensite phase being smaller than the EBSD step and probe sizes. The Pphase precipitates are on the order of $100 \mathrm{~nm}$ with the matrix phase between the precipitates being smaller. ${ }^{7}$ Though this is unfortunate for studying the SMA-microstructure behavior, the mapping successfully identified a large single grain, the turquois colored grain oriented near the $\langle 145\rangle$ direction (Euler angles: $\varphi_{1}=12^{\circ}, \theta=38.5^{\circ}, \varphi_{2}=198^{\circ}$ ) in the top left of the map, that was suitable for machining an array of micropillars

\section{Microcompression Testing}

Microcompression testing was first performed on a series of pillars at room temperature to determine both the consistency of the measurements and the cyclic behavior of the alloy in the low temperature phase condition.

Stress-strain behavior at ambient temperature (Fig. 3a) was observed to be very consistent between the four different micropillars, which were compressed to varying levels of strain. Some variation was observed after the onset of general yielding; however, this is attributed to the presence of $\mathrm{TiC}$ particles, as seen in Fig. 3b as micron-sized darker regions. The slip traces observable after deformation are irregular (Fig. 3c), rather than the straight slip traces that are typical in deformed single crystal micropillars. ${ }^{15}$ This type of behavior was observed in plastically deformed pillars at all temperatures (see Fig. 4, below). The determination of the slip planes was attempted by analyzing the slip traces visible on the sidewalls of the pillars. The slip traces have a jagged appearance. For a number of
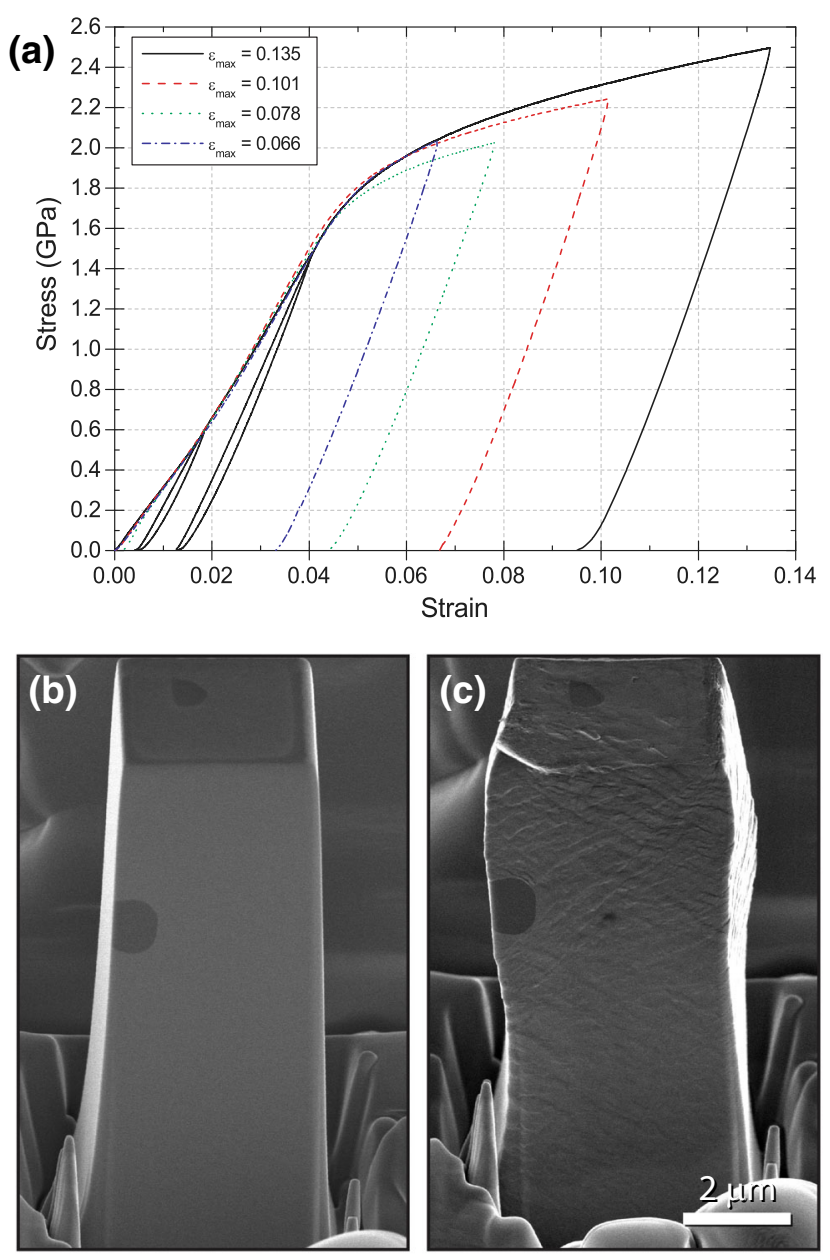

Fig. 3. (a) Engineering stress-strain behavior at $24^{\circ} \mathrm{C}$ and high resolution SE micrographs, (b) before and (c) after compression showing irregular slip lines characteristic of pencil glide and darker TiC particles. 

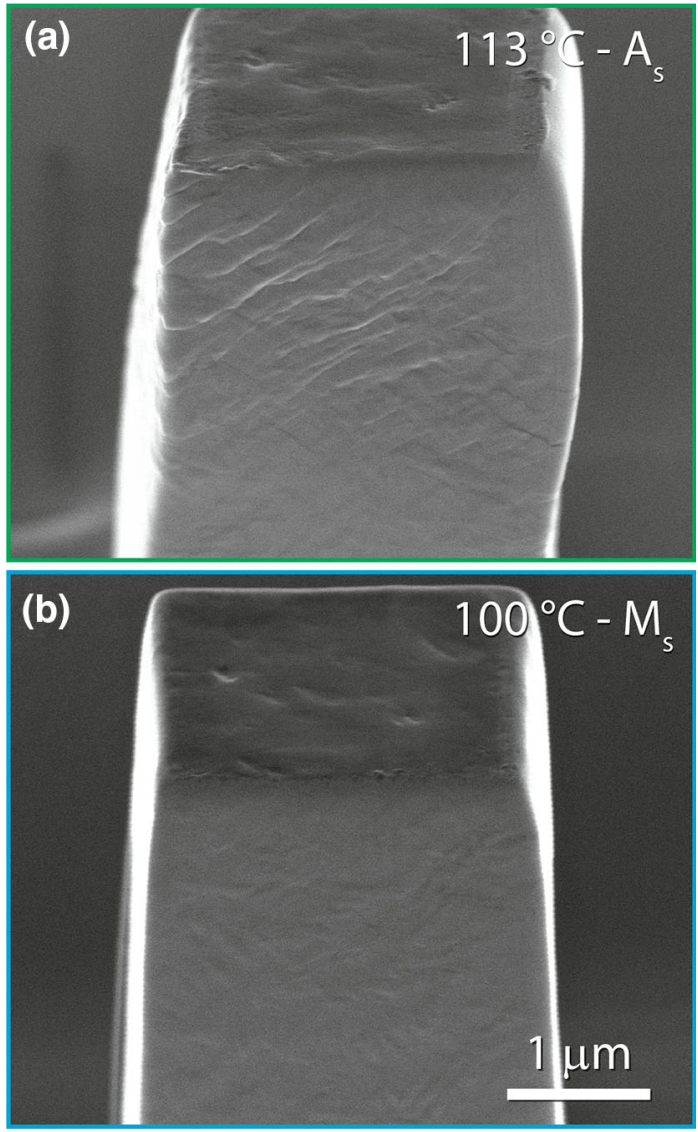

Fig. 4. Secondary electron micrographs of pillars compressed to (a) 0.08 applied strain at $113^{\circ} \mathrm{C}$ and (b) 0.04 applied strain at $100^{\circ} \mathrm{C}$

straight segments of the jagged slip trace, their crystallographic directions have been determined with the help of the grain orientation which is known due to the EBSD analysis. This is done by measuring the angles of the slip traces from SE micrographs taken at known angles to the EBSDdetermined grain orientation. Since the crystal directions of the slip trace segments must be perpendicular to the according slip plane normal, one can determine possible slip planes. In this way, it was inferred that slip occurred on planes of the families $\{110\},\{111\}$ and $\{112\}$. These determined slip planes are also the ones that can be expected in the case of pencil glide. The presence of all these slip planes in one pillar suggest that slip occurred via pencil glide around the precipitates, which is in perfect accordance with the jagged appearance of the slip traces.

Stress-strain behavior at elevated temperatures was investigated using multi-cycle load-unloadreload microcompression testing (Fig. 5). During elevated temperature compression above the austenite start temperature, the loading slope was observed to double in stiffness, corresponding to the increasing stiffness of the B2 phase. This reversed back to the austenite stiffness upon cooling below the martensite start temperature (Fig. 5b). Due to the overlapping loading cycles, it is difficult to directly observe the differences in the stress-strain behavior at different temperatures. To clarify this, the first cycle from each temperature is shown in Fig. 6.

Upon heating (Fig. 6a), the characteristic 'flagshape' for the pseudoelastic behavior in the single crystal was evident. This characteristic pseudoelastic behavior is observed to peak at the austenite start temperature. The observed recovery is not complete, as seen previously ${ }^{8}$ in grains more closely oriented to the $\langle 101\rangle$ plane. This may be due to the crystal orientation increasing the Schmid factor and favoring slip past the phase transformation temperature; however, the observed pencil glide shows that multiple slip systems are being activated simultaneously, as evidenced by the multiple slip traces in Fig. 3c. This suggests that the small (a)

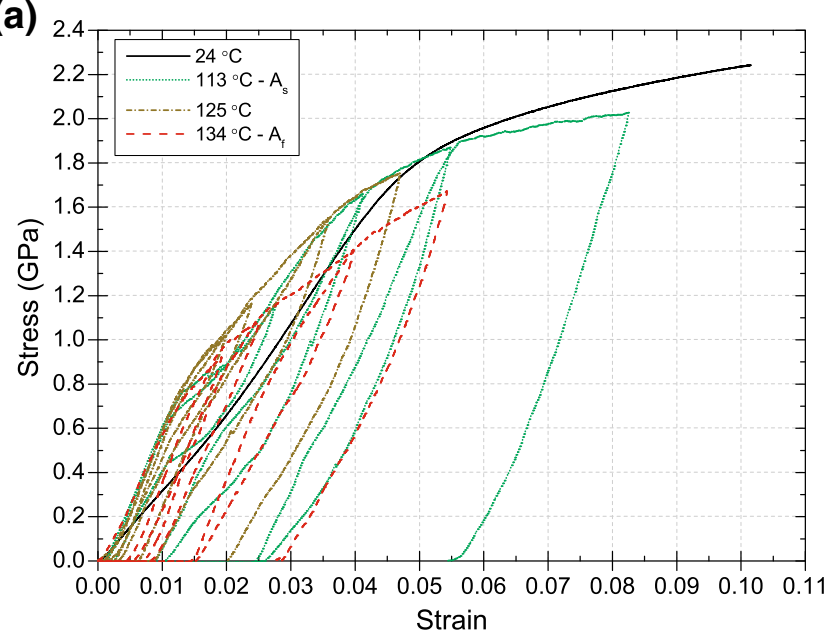

(b)

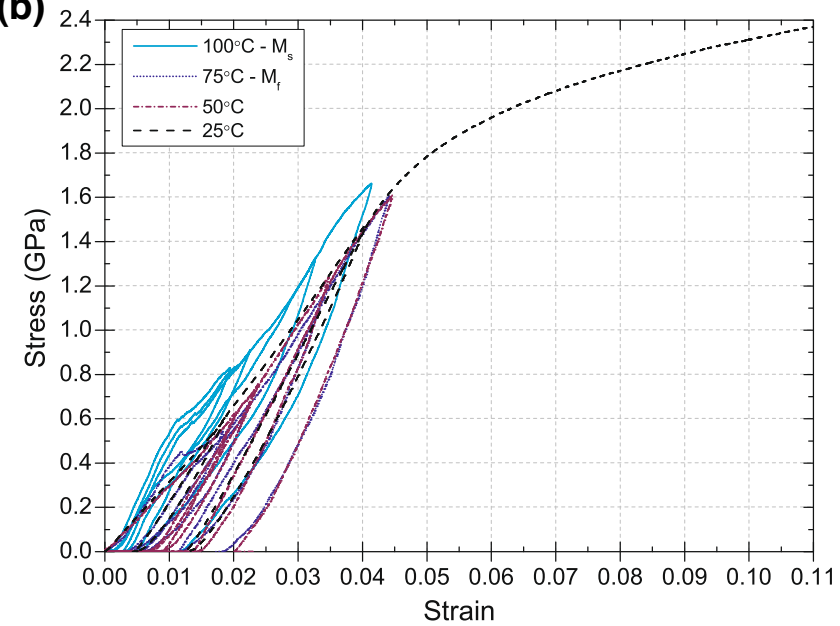

Fig. 5. Engineering stress-strain relationships obtained from multi-cycle microcompression at various temperatures during (a) heating and (b) cooling. 

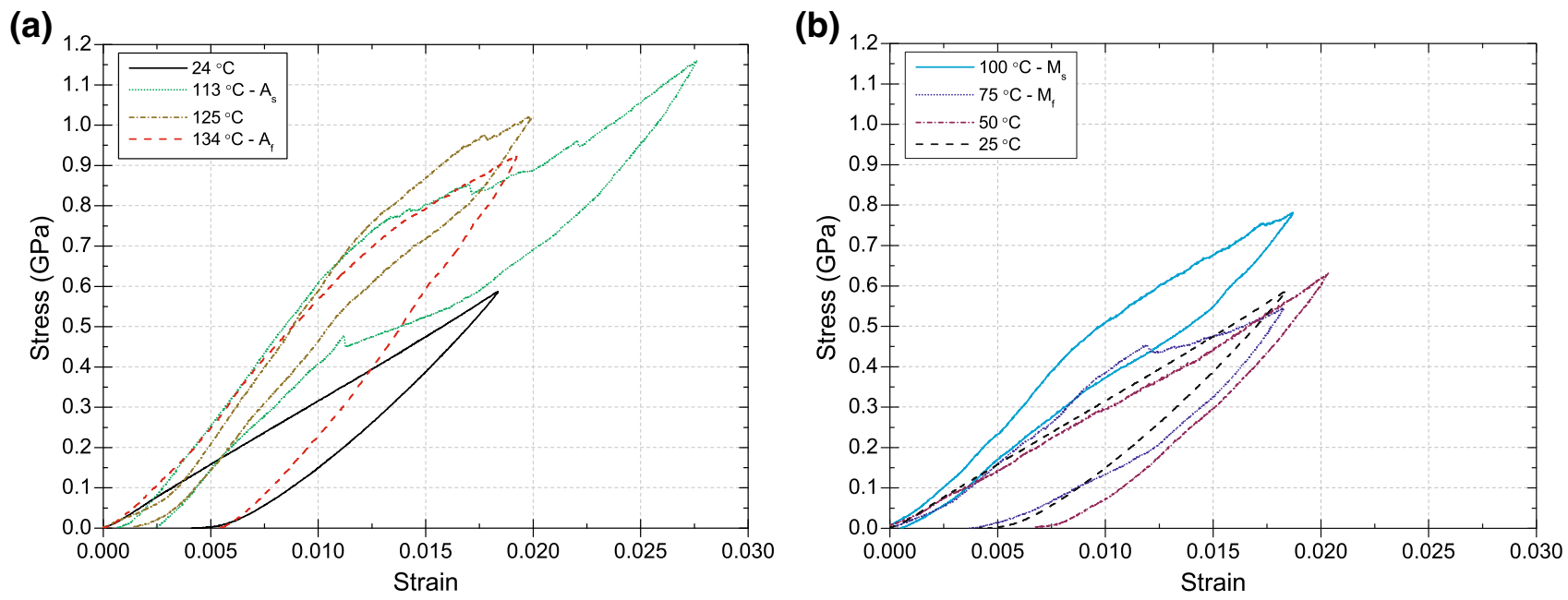

Fig. 6. Engineering stress-strain relationships from the first load-unloading cycle at various temperatures during (a) heating and (b) cooling.

amount of plasticity is likely contributing to misalignment between the flat punch and the micropillar resulting in stress concentrations and deformation at the corners of the pillars. Some stress concentrations are also expected around the TiC particles, which may account for additional plasticity. Regardless of these mitigating factors, the superelastic recovery of the pillars at $113^{\circ} \mathrm{C}$ and $125^{\circ} \mathrm{C}$ at low applied strains is nearly complete. By the austenite finish temperature, little evidence of pseudoelastic recovery can be observed during unloading. During cooling, pseudoelastic behavior is only observed at the martensite start temperature. Below this temperature, deformation is again purely plastic.

Upon examination of the pillars deformed at elevated temperatures, the same pencil glide morphology of slip observed at ambient temperature in Fig. $3 \mathrm{c}$ is also observable in the heavily strained pillar (Fig. 4a). If the pillars are only slightly strained to slightly beyond the pseudoelastic regime (Fig. 4b), then the martensite lath precipitates become more visible due to deformation of the surrounding matrix.

Finally, one of the major benefits of this study is that a comparison between the single (oriented) grain behavior can be compared to its polycrystalline pseudoelastic behavior as reported by Sasaki et al. ${ }^{7}$ To quantify the relationship between the applied strain and the pseudoelastic recovery, the remnant strain ratio after unloading was determined from each load-unload cycle as a function of temperature (Fig. 7). The remnant strain was calculated as the median between the strain where the stress drops to zero on unloading from the previous cycle and the strain where the stress first begins to rise on the following cycle. What is apparent is that the polycrystalline sample is able to have at least an order of magnitude reduction in remnant strain than that of the single crystal. This is attributed to

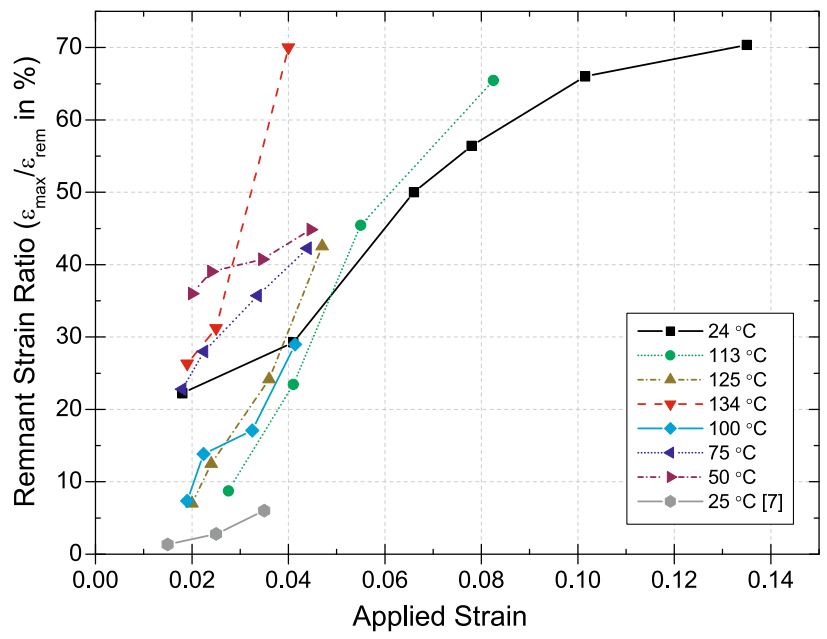

Fig. 7. Remnant strain ratio after unloading during multi-cycle microcompression as a function of applied strain with a comparison to bulk values acquired previously ${ }^{7}$.

deformation being accommodated by multiple grains, each with different slip systems that can more readily be activated under the loading orientation. This allows small amounts of strain to be accommodated primarily by grains which are ideally oriented for the martensitic transformation, whereas in single crystals the crystal orientation is fixed at a value which may or may not be ideal.

In order to consider the limits of the pseudoelastic behavior, a criterion for pseudoelasticity must first be specified. In this case, we designate pseudoelastic behavior to be indicated when the remnant strain ratio falls below the behavior observed at room temperature. This criterion would be inappropriate if the material's modulus decreased during the martensitic transformation, since this would cause the remnant strain ratio to decrease as a function of the applied strain even if the material had similar 
plastic behavior. However, in this case, the materials modulus increases on the martensitic transformation, so the amount of recovered applied strain due to normal elasticity would decrease. This means that any additional recovered strain, or a decrease in the remnant strain ratio, would imply that pseudoelasticity is operating. This suggestion is supported by the curve for $134^{\circ} \mathrm{C}$ in Fig. 7 , where all martensite precipitates should be fully in the austenite phase, having a higher remnant strain ratio than the room temperature curve at all applied strains.

Using this criterion, some pseudoelasticity is indicated in the stress-strain behavior from pillars at three different temperatures, corresponding to the austenite start, austenite peak, and martensite start. Figure 7 shows that the highest applied strain to retain pseudoelastic behavior was achieved at the austenite start temperature, $113^{\circ} \mathrm{C}$, which intersects room temperature behavior until an applied strain of 0.049 . The martensite start temperature was slightly less at 0.041 applied strain, and the austenite peak temperature was only slightly less than that at 0.038 applied strain. Of course, a more desirable criterion for perfect pseudoelasticity would be a remnant strain ratio of $1-2 \%$, as observed in bulk, but this was not achievable with the crystal orientations available for this work. Instead, the criterion used here simply indicates whether any pseudoelastic contribution to the recovery can be observed.

\section{CONCLUSION}

To summarize our findings, microthermomechanical testing has been successfully applied to investigate the elevated temperature pseudoelasticity and deformation of a Ni-Ti-Pd alloy. Elevated temperature EBSD confirmed that phase transformations occur only at a submicron length scale consistent with the fine precipitates previously observed. $^{7}$ Pseudoelasticity was successfully observed at elevated temperatures and compared to bulk polycrystalline behavior. It was found that a polycrystalline sample, due to the numerous grains, is able to accommodate the deformation and exhibit better pseudoelastic recovery. In contrast, a single grain accommodated all the deformation, and plasticity was observed to occur via pencil glide, presumably around the fine scale precipitates.

\section{ACKNOWLEDGEMENTS}

MLW and GBT acknowledge the NASA grant NNX09AO61A and the NASA FAP Supersonics project, Dale Hopkins, API. The authors would like to thank D. Frey \& G. Buerki for technical assistance with the in situ SEM Indenter.

\section{REFERENCES}

1. P.G. Lindquist and C.M. Wayman, Shape Memory and Transformation Behavior of Martensitic Ti-Pd-Ni and Ti-Pt$\mathrm{Ni}$ Alloys.Engineering Aspects of Shape Memory Alloys, ed. T.W. Duerig, K.N. Melton, D. Stöckel, and C.M. Wayman (London: Butterworth-Heinemann, 1990).

2. J. Ma, I. Karaman, and R.D. Noebe, Int. Mater. Rev. 55, 257 (2010).

3. G.S. Bigelow, S.A. Padula, A. Garg, D. Gaydosh, and R.D. Noebe, Metall. Mater. Trans. A 41, 3065 (2010).

4. H. Hosoda, S. Hanada, K. Inoue, T. Fukui, Y. Mishima, and T. Suzuki, Intermetallics 6, 291 (1998).

5. R. Noebe, I. Santo Padula, G. Bigelow, O. Rios, A. Garg, and B. Lerch, Properties of a Ni19. 5Pd30Ti50. 5 High-Temperature Shape Memory Alloy in Tension and Compression. Smart Structures and Materials (London: International Society for Optics and Photonics, 2006), p. 617010.

6. L. Kovarik, F. Yang, A. Garg, D. Diercks, M. Kaufman, R. Noebe, and M. Mills, Acta Mater. 58, 4660 (2010).

7. T.T. Sasaki, B.C. Hornbuckle, R.D. Noebe, G.S. Bigelow, M.L. Weaver, and G.B. Thompson, Metall. Mater. Trans. A 44, 1388 (2013).

8. J. Pfetzing-Micklich, R. Ghisleni, T. Simon, C. Somsen, J. Michler, and G. Eggeler, Mater. Sci. Eng. A 538, 265 (2012).

9. D.M. Norfleet, P.M. Sarosi, S. Manchiraju, M.F.X. Wagner, M.D. Uchic, P.M. Anderson, and M.J. Mills, Acta Mater. 57, 3549 (2009).

10. J.M. Wheeler, D.E.J. Armstrong, W. Heinz, and R. Schwaiger, Curr. Opin. Solid State Mater. Sci. (2015). doi:10. 1016/j.cossms.2015.02.002.

11. J.M. Wheeler and J. Michler, Rev. Sci. Instrum. 84, 045103 (2013).

12. J.M. Wheeler, P. Brodard, and J. Michler, Philos. Mag. 92, 3128 (2012).

13. S. Korte and W.J. Clegg, Scripta Mater. 60, 807 (2009).

14. J.M. Wheeler, R. Raghavan, and J. Michler, Mater. Sci. Eng. A 528, 8750 (2011).

15. J. Wehrs, G. Mohanty, G. Guillonneau, A.A. Taylor, X. Maeder, D. Frey, L. Philippe, S. Mischler, J.M. Wheeler, and J. Michler, JOM 67, 1684 (2015). 